\title{
Expresión de ICAM-1 en el Endotelio de Arterias Humanas Mediante Inmunohistoquímica
}

\author{
ICAM-1 Expression in Endothelium of Human Arteries by Immunohistochemistry
}

\author{
"Rodrigo Moore-Carrasco; "*Wendy Donoso; "Francisca Sutin; "Macarena Vergara; "Lorena Toloza; *Jaime Gonzalez; \\ ***,*****Erik Morales \& *Iván Palomo
}

MOORE-CARRASCO, R.; DONOSO, W.; SUTIN, F.; VERGARA, M.; TOLOZA, L.; GONZALEZ, J.; MORALES, E. \& PALOMO, I. Expresión de ICAM-1 en el endotelio de arterias humanas mediante inmunohistoquímica. Int. J. Morphol., 29(4):1351-1356, 2011.

RESUMEN: Las enfermedades cardiovasculares son la principal causa de muerte a nivel mundial. Entre ellas tienen gran relevancia las de tipo isquémicas, en donde el desarrollo de placas ateroscleróticas es el proceso fisiopatológico central. El estudio de la aterosclerosis es fundamental para comprender como se inicia este proceso patológico y los factores que influyen en su desarrollo. Distintas metodologías de laboratorio, entre otras la inmunohistoquímica, permiten reconocer las células y moléculas que participan en el proceso ateromatoso y que van interactuando según la progresión de la lesión. Un marcador de disfunción endotelial es la mayor expresión de la molécula de adhesión intercelular ICAM-1. En este trabajo se realizó la estandarización de inmunohistoquímica para la molécula de adhesión ICAM-1, y se estudió su expresión en arterias humanas sanas y con placa ateromatosa. En las muestras de arterias humanas con patología aterosclerótica, la expresión de ICAM-1 se observó aumentada, pero fue de difícil reconocimiento. Esto principalmente porque el tejido empleado como control en la estandarización fue una amígdala con hiperplasia y proceso inflamatorio que aumenta notablemente la expresión de ICAM-1. La implementación del método de inmunohistoquímica para ICAM-1 en arterias humanas permitirá conocer estados de disfunción endotelial y el desarrollo futuro del diseño e implementación de métodos de diagnóstico en aquellos procesos ateroclerótico en estado incipiente.

PALABRAS CLAVE: ICAM-1; Aterosclerosis; Inmunohistoquímica.

\section{INTRODUCCIÓN}

Las enfermedades cardiovasculares (ECV) son la principal causa de mortalidad en el mundo (Balkau et al., 2002). En una gran cantidad de casos, por tratarse de eventos trombóticos arteriales, tienen como proceso previo la aterosclerosis, la cual es una de las principales consecuencias producidas por la asociación de los llamados "factores de riesgo cardiovascular" (obesidad, hipertensión arterial, diabetes y dislipidemias) (Bustos et al., 2003; Dandona et al., 2005; Palomo et al., 2006).

La aterosclerosis es una enfermedad inflamatoria crónica que se caracteriza por acumulación progresiva de material graso en la capa íntima de la pared arterial concomitantemente con el crecimiento de la capa muscular lisa y una respuesta inflamatoria localizada (Calderón et al.,
2008). Este proceso inflamatorio conlleva a una serie de interacciones moleculares, principalmente entre endotelio, plaquetas y leucocitos (Cybulski \& Gimbrone, 1991). En dichas interacciones participan varias moléculas de adhesión y glicoproteínas de membrana. Entre las moléculas de adhesión se encuentran la superfamilia de las inmunoglobulinas VCAM (vascular cell adhesion molecule) e ICAM-I (Inter-Cellular Adhesion Molecule 1), y la familia de las Selectinas (E-selectina y P-selectina) (Cobb et al., 1996). ICAM-1 es una molécula que se expresa en las membranas de los leucocitos y de las células endoteliales. Las personas con riesgo cardiovascular, como es el caso de quienes presentan Síndrome Metabólico (SM), presentan niveles séricos elevados de factor de necrosis tumoral alfa (TNF $\alpha$ ) e interleuquina-1 (IL-1), citoquinas inflamatorias

* Programa de Investigación en Factores de Riesgo de Enfermedades Cardiovasculares (PIFRECV), Departamento de Bioquímica Clínica e Inmunohematología, Facultad de Ciencias de la Salud, Universidad de Talca, Talca, Chile.

** Unidad de Patología, Facultad de Ciencias de la Salud, Universidad de Talca, Talca, Chile.

*** Escuela de Medicina, Universidad Católica del Maule, Talca, Chile.

***** Unidad de Anatomía Patológica, Hospital Regional de Talca, Talca, Chile.

Este estudio ha sido financiado por FONDECYT INICIACIÓN N 11090027 
que promueven la expresión de ICAM-1 (Wertheimer et al., 1992; Palomo et al., 2009; Palomo et al., 2010).

El propósito de este estudio, luego de estandarizar el método de inmunohistoquímica para ICAM-1, fue evidenciar su expresión en arterias humanas.

\section{MATERIAL Y MÉTODO}

Estandarización para ICAM-1 por inmuno-histoquímica en tonsila humana. Como primera etapa de estandarización del método de inmunohistoquímica para ICAM-1, se utilizó una tonsila palatina humana hiperplásica en proceso inflamatorio latente. La muestra fue fijada en formaldehído $4 \%$ en tampón fosfato de sodio $0,075 \mathrm{M} \mathrm{pH} 7,3$. Posteriormente se realizaron cortes de $5 \mu \mathrm{m}$ de espesor en portaobjetos silanizados con 3-aminopropyltriethoxysilano $2 \%$ en acetona, para inmuno-histoquímica y para tinción convencional hematoxilina-eosina. Estos se desparafinaron y rehidrataron siguiendo el protocolo de rutina del Laboratorio de Histopatología de la Universidad de Talca (Kiernan, 2008).

Para el análisis inmunohistoquímico se utilizó un anticuerpo primario monoclonal anti-ICAM-1 humano (Novocastra, UK) y para la recuperación de antígenos, se usó solución tampón citrato de sodio $0,01 \mathrm{M}$ pH 6 , en vaporera. Como bloqueador de peroxidasas endógenas se utilizó peróxido de hidrógeno 3\%, junto a un sistema de detección Level 2 $\mathrm{USA}^{\mathrm{TM}}$, Ultra Streptavidin Detection Systems (Signet, USA) y como cromógeno revelador ImmPACT ${ }^{\mathrm{TM}}$ DAB Peroxidase Substrate (Vector, USA). El sistema de detección y el cromógeno fueron utilizados de acuerdo al protocolo indicado por el fabricante. Se realizó contraste nuclear con hematoxilina.

Las variables que se consideraron en la estandarización del método fueron concentración (1/10, 1/25, 1/50 y 1/100), tiempo (30 min y $1 \mathrm{hr}$ ) y temperatura de incubación $\left(25^{\circ} \mathrm{C}\right.$ y $37^{\circ} \mathrm{C}$ ) del anticuerpo primario. Como controles negativos se utilizó cortes de tejido de mucosa oral normal y un control negativo universal en que se omitió el anticuerpo primario. Paralelamente se realizaron cortes de $5 \mu \mathrm{m}$ para realizar tinción hematoxilina eosina.

Expresión de ICAM-1 en endotelio de arteria de conejo. Con el propósito de contar con un control positivo atingente al estudio realizado, es que mediante inducción química en arteria de conejo de obtuvo un tejido acorde a la expresión requerida para ICAM-1. Para ello, se utilizó conejo neozelandes, desde el cual, posterior a ser anestesiado, se disecó quirúrgicamente la arteria carótida en la que se indujo la trombosis mediante la aplicación de cloruro férrico 10\% (Kurz et al., 1990). Durante el tiempo que duró el procedimiento el animal fue monitoreado mediante Detector de flujo Doppler modelo 811-B (Parks Medical Electronics, USA). Finalmente se extrajeron ambas carótidas las que fueron fijadas en formaldehído $4 \%$ en tampón fosfato de sodio $0,075 \mathrm{M} \mathrm{pH} 7,3$ para su posterior procesamiento histológico.

Expresión de ICAM-1 en endotelio de arterias humanas. Para el análisis de la expresión de ICAM-1 en endotelio de arterias humanas se utilizó una arteria sana (aorta), dos con lesión ateromatosa en distintos estados de desarrollo (arterias carótida y poplítea). Los criterios para la clasificación de las arterias estudiadas con proceso de aterosclerosis, se aplicaron según la American Heart Association (AHA) (Stary et al., 1995; Lovett et al., 2004).

Las muestras para estandarización y estudio fueron tomadas desde el material de archivo del Hospital Regional de Talca.

\section{RESULTADOS}

\section{Estandarización para ICAM-1 por inmuno- histoquímica en tonsila palatina humana}

La figura $1 \mathrm{~A}$ y B muestra cortes de la tonsila palatina teñidos con hematoxilina- eosina. La superficie y las criptas tonsilares con revestimiento epitelial pluriestratificado pavimentoso no queratinizado característico de este tipo de tejido. A nivel subyacente destaca el tejido linfoide difuso y nodular con predominio de los folículos secundarios con formación de centros germinales. En la figura 1C, con aumento mayor (40x), se muestra la porción de un centro germinal de un folículo linfoide. En la figura 1D, los folículos linfoides muestran una zona externa densa constituida por linfocitos de pequeño tamaño que constituyen el manto y un centro claro con población celular heterogénea. Cuando el anticuerpo primario se diluyó $1 / 10$ y la incubación se realizó a $25^{\circ} \mathrm{C}$ se observó positividad moderada a intensa en gran parte de los linfocitos foliculares y en la zona del manto, junto con el endotelio arteriolar. Además se observan focos de marcación inespecífica e intersticial. Por su parte, en la muestra incubada a $37^{\circ} \mathrm{C}$, se observó una reactividad intensa, principalmente a nivel de membrana en la mayoría de los linfocitos foliculares y zona del manto, se aprecian zonas de marcación inespecífica.

$\mathrm{Al}$ diluir el anticuerpo primario $1 / 25$ e incubar a $25^{\circ} \mathrm{C}$ (figura $1 \mathrm{E}$ ) se observó positividad predominantemente a nivel de membrana con moderada a intensa marcación, en la mayoría de los linfocitos foliculares y adyacentes a criptas a nivel de membrana, además del endotelio arteriolar. 


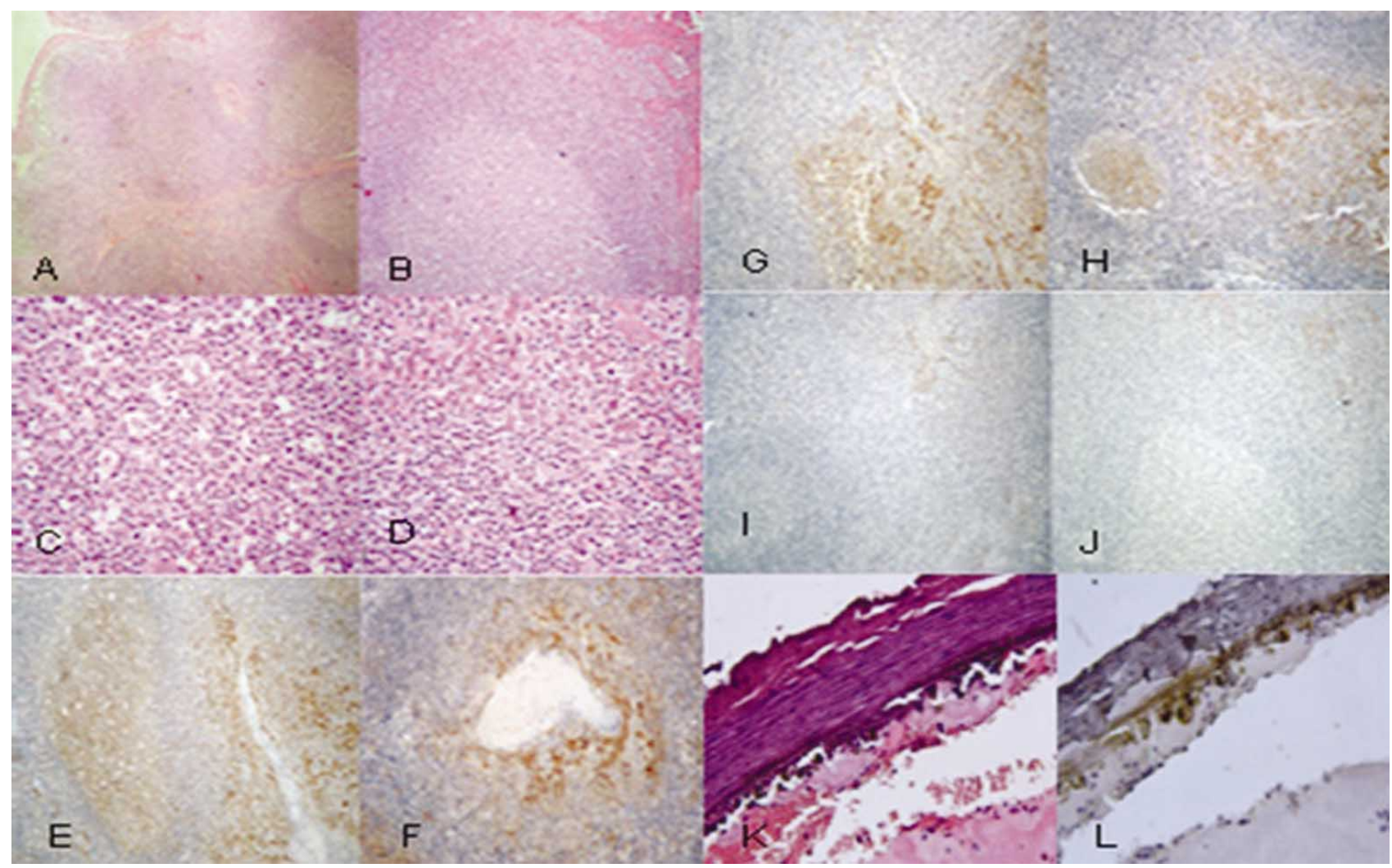

Fig. 1. Tonsila palatina hiperplásica. Tinción hematoxilina eosina A (4x), B (10x), C, D(40x). Inmunohistoquímica concentración $1 / 25$ reactividad a $25^{\circ} \mathrm{C} \mathrm{E}(40 \mathrm{x})$ y a $37^{\circ} \mathrm{C} \mathrm{F}(40 \mathrm{x})$; concentración $1 / 50$ reactividad a $25^{\circ} \mathrm{C} \mathrm{G}(10 \mathrm{x})$ y a $37^{\circ} \mathrm{C} \mathrm{H}(10 \mathrm{x})$; concentración $1 / 100$ reactividad a $25^{\circ} \mathrm{C} \mathrm{I}(10 \mathrm{x})$ y a $37^{\circ} \mathrm{C} \mathrm{J}(10 \mathrm{x})$. Arteria carótida de conejo hematoxilina eosina $\mathrm{K}(40 \mathrm{x})$ e inmunohistoquímica L (40x)

En la muestra incubada a $37^{\circ} \mathrm{C}$ (Fig. $\left.1 \mathrm{~F}\right)$, se apreció una positividad intensa, principalmente de membrana y en la mayor parte de los linfocitos adyacentes a criptas. Focalmente se percibe intensa positividad en linfocitos foliculares y paredes arteriolares.

Cuando el anticuerpo primario fue diluido $1 / 50$ y la reacción incubada a $25^{\circ} \mathrm{C}$ y $37^{\circ} \mathrm{C}$ se apreció positividad predominantemente de membrana con una marcación moderada a intensa en la mayoría de los linfocitos adyacentes a criptas (Fig. 1 G-H). Además se observó focalmente, moderada positividad en linfocitos foliculares.

Al diluir 1/100 el anticuerpo anti-ICAM-1, a ambas temperaturas se observó reactividad leve, predominantemente de membrana, en escasos linfocitos subepiteliales reconocidos a nivel de criptas (Fig. 1 I-J).

Dado los resultados obtenidos en la estandarización, para el estudio inmunohistoquímico en las arterias estudiadas se utilizó la concentración $1 / 25$ e incubación a $37^{\circ} \mathrm{C}$, para el anticuerpo primario.

Las imágenes $\mathrm{K}$ y L corresponden a cortes histológicos de pared arterial de conejo. Figura 1K (40x) se observa pared arterial de histoarquitectura conservada. A nivel de capa íntima destaca superficie de revestimiento endotelial irregular, asociada a cúmulos de material eosinófilo-fibrinoso, corpúsculos granulares y algunas células inflamatorias. En figura 1L se observa intensa positividad endotelial para inmunomarcación con ICAM-1, además de marcación en células inflamatorias.

\section{ICAM-1 en arterias humanas sanas y con proceso aterosclerótico}

a) ICAM-1 en arteria aorta humana sana. Los cortes de pared de arteria aorta humana sana teñidos con hematoxilinaeosina, observada con distintos aumentos (Fig. 2 A-C), muestran tres túnicas, destacando predominio de la media, la que se aprecia rica en fibras elásticas. La íntima muestra endotelio conservado y en la adventicia se aprecia conjuntivo laxo y vasa vasorum. En la figura $2 \mathrm{~B}$ destaca el escaso espesor de la túnica íntima, al igual que en la figura $2 \mathrm{C}$, con endotelio aplanado y una adventicia compuesta de conjuntivo laxo, incluyendo elementos de vasa vasorum.

En la inmunohistoquímica para ICAM-1 en las mismas arterias (Fig. $2 \mathrm{~J}$ y K) se observa ausencia de inmunorreactividad. 


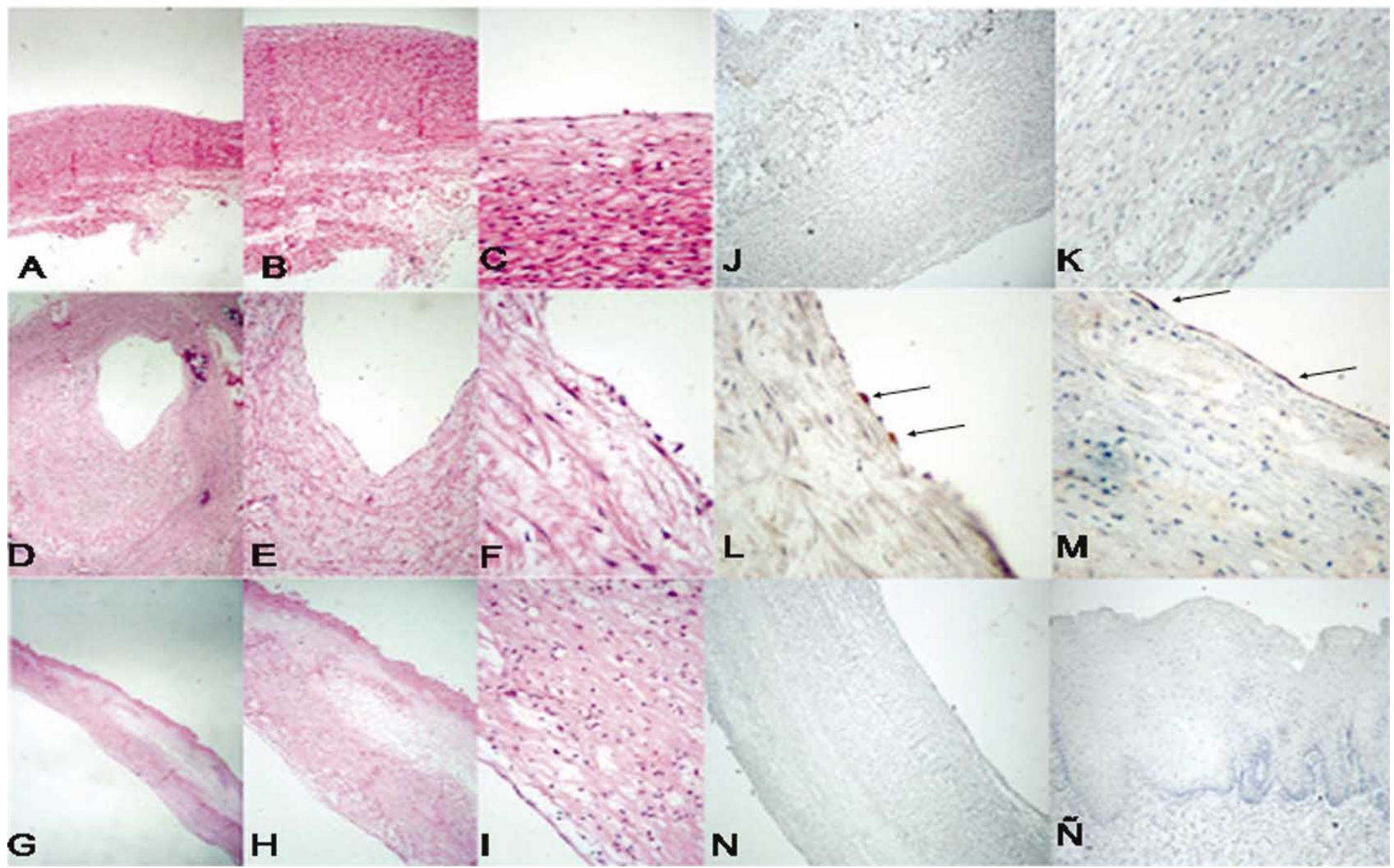

Fig. 2. Arterias humanas. Tinción hematoxilina eosina arteria: aorta sana $\mathrm{A}(4 \mathrm{x}),(10 \mathrm{x})$ y $\mathrm{C}(40 \mathrm{x})$; arteria poplítea $\mathrm{D}(4 \mathrm{x}), \mathrm{E}(10 \mathrm{x})$ y $\mathrm{F}(40 \mathrm{x})$; arteria carótida $\mathrm{G}(4 \mathrm{x}), \mathrm{H}(10 \mathrm{x})$ y I(40x). Inmunohistoquímica: arteria aorta sana J(10x) y K(40x); arteria poplítea L(40x); arteria carótida $\mathrm{M}(40 \mathrm{x})$; controles negativos: arteria carótida $\mathrm{N}(10 \mathrm{x})$ y mucosa oral normal $\tilde{\mathrm{N}}$ (10x).

b) ICAM-1 en arteria poplítea humana con placa ateromatosa. La figura $2 \mathrm{E}-\mathrm{F}$ muestra cortes histológicos de pared arterial de tipo muscular, teñidos con hematoxilinaeosina, con desarrollo de lesión ateromatosa compatible con tipo $\mathrm{V}$ (placa calcificada) según la clasificación de American Heart Association (AHA) y "probablemente inestable" según la clasificación de Lovett (Lovett et al., 2004). En figura $2 \mathrm{D}$ se observa íntima engrosada, con material amorfo extracelular, abundante infiltrado de macrófagos de citoplasma espumoso, proliferación fibroblástica y moderado infiltrado linfocitario. Túnica media con abundantes fibras musculares lisas y focos de calcificación distrófica. En la figura $2 \mathrm{E}$ y $\mathrm{F}$, se aprecia, el engrosamiento subendotelial que incluye proliferación fibroblástica e infiltrado linfocitario.

La inmunohistoquímica destaca una leve positividad del endotelio a la inmunomarcación con ICAM-1, predominantemente de membrana (Fig. $2 \mathrm{~L}$ ).

c) ICAM-1 en arteria carótida humana con placa ateromatosa. La figura $2 \mathrm{G}-\mathrm{I}$ muestra cortes histológicos de pared arterial de tipo muscular, teñidos con hematoxilinaeosina, con desarrollo de lesión ateromatosa compatible con tipo IV (ateroma) según la clasificación de AHA y "probablemente inestable" según la clasificación de Lovett (Lovett et al.). En figura $2 \mathrm{G}-\mathrm{H}$ se observa porción de túnicas media e íntima con moderada a intensa distorsión de la arquitectura. Se observa cúmulo de material amorfo extracelular, presencia de focos de macrófagos de citoplasma espumoso, escaso infiltrado linfocitario y focos de exudación polimorfonuclear. En la figura 2 I, se aprecia además incipiente fibroblástica y un revestimiento endotelial conservado.

En la figura $2 \mathrm{M}$, la inmunohistoquímica muestra leve a moderada positividad del endotelio a la inmunomarcación con anticuerpo anti ICAM-1, predominantemente de membrana.

d) Controles para inmunohistoquímica en arterias humanas. Como control negativo se utilizó cortes de dos tipos de tejido. El primero corresponde a una muestra de arteria carótida patológica en que se omitió el anticuerpo primario, presentando inmunorreactividad negativa (figura $2 \mathrm{~N}$ ) y otro correspondiente a tejido de mucosa oral sana que fue tratada en iguales condiciones que las muestras en estudio, presentando inmunoreactividad negativa para ICAM-1 (figura $2 \tilde{\mathrm{N}}$ ). 


\section{DISCUSIÓN}

La aterosclerosis es una enfermedad inflamatoria, en donde las lesiones producidas corresponden a una serie de respuestas celulares y moleculares a este proceso inflamatorio (Badimon et al., 2009). Una etapa temprana en el proceso aterosclerótico es el desarrollo de la disfunción endotelial, caracterizada entre otros aspectos, por la expresión creciente de moléculas de adhesión celular, como VCAM-I, E-Selectina e ICAM-1 (Cybulski \& Gimbrone; Viganò et al., 2003) siendo la pesquisa de esta última a través de inmunohistoquímica el objetivo en este trabajo.

Las técnicas inmunohistoquímicas representan un apoyo relevante para el estudio de ciertos marcadores de disfunción endotelial, al permitir revelar la ubicación y junto a ello la expresión de proteínas específicas en el tejido, sin perder el punto de referencia que resulta muy relevante en la comprensión de eventos fisiológicos normales y patológicos. Sin embargo, ésta es una técnica en la cual diversos factores pueden afectar su estandarización; la manipulación de los cortes y el procedimiento debe ser cuidadoso (Delgado-Gonzáles $\&$ Leyva-Sartori, 2006). Es por ello que en una primera etapa, se estandarizó una técnica de inmunohistoquímica para ICAM1 en cortes de tonsila humana hiperplásica con un proceso inflamatorio latente. Luego de modificar las concentraciones, temperaturas y tiempos de incubación para el anticuerpo primario, se determinó que la concentración $1 / 25$ a $37^{\circ} \mathrm{C}$ por 30 minutos resulta óptima en término de inmunomarcación específica y bajo nivel de tinción de fondo "background", concentración que se ajusta a lo sugerido por el fabricante del anticuerpo monoclonal.

Posteriormente se trabajó en poner de manifiesto la expresión de esta molécula de adhesión en arterias humanas con proceso aterosclerótico, en distintos estados de avance. Se comprobó lo señalado por algunos investigadores en relación a la sobreexpresión de la mencionada ICAM-1 en eventos que implican injuria endotelial (Chatterjee, 1998); se ha planteado que esta molécula de adhesión es un marcador temprano en aterosclerosis (Lehmann et al., 2003). Esto último dado principalmente por la expresión de ICAM-1 en la arteria carótida con un estado incipiente aterosclerótico.

ICAM-1 se expresa en las células endoteliales activadas, en respuesta a citoquinas inflamatorias como IL-1 $\beta$ y TNF- $\alpha$ de forma paralela a la migración leucocitaria (Velarde et al., 2007).

En la arteria poplítea estudiada que presentaba estado más avanzado de aterosclerosis, dado principalmente por el estado de calcificación distrófica, entre otras característi- cas histológicas, la expresión de ICAM-1 se apreció ligeramente disminuida en relación al estado incipiente que presentó la arteria carótida; este hecho podría estar implicando el rol de ICAM-1 en la trasmigración de los leucocitos en el endotelio vascular en procesos tales como la extravasación y la respuesta inflamatoria produciéndose por la interacción leucocito-endotelio (Janeway et al., 2004).

Al comparar la expresión de ICAM-1 entre tejidos sanos y patológicos (en distintos estados de avance), se confirma lo observado por otros autores en el sentido que esta molécula de adhesión se encuentra sobreexpresada en los procesos inflamatorios ateroscleróticos (Lehmann et al.; Fuster \& Sanz, 2005).

Contar con el método de inmunohistoquímica para ICAM-1 en arterias fijadas en formol y embebidas en parafina, permitirá a nuestro grupo de investigación estudiar estados de disfunción endotelial en animales de experimentación sometidos a diferentes intervenciones, que a futuro permitan aportar datos relevantes para el diseño e implementación de métodos de diagnóstico precoz de procesos ateroscleróticos.

MOORE-CARRASCO, R.; DONOSO, W.; SUTIN, F.; VERGARA, M.; TOLOZA, L.; GONZALEZ, J.; MORALES, E. \& PALOMO, I. ICAM-1 expression in endothelium of human arteries by immunohistochemistry. Int. J. Morphol., 29(4):13511356, 2011.

SUMMARY: Cardiovascular diseases (CVD) are the leading cause of death in the world. Among them the ischemic type are of great importance, where the development of atherosclerotic plaques is the central pathophysiological process. The study of atherosclerosis is critical to understand how this disease process begins and factors influencing its development. Various laboratory methods, including immunohistochemistry, allow the recognition of cells and molecules involved in the atheromatous process that are interacting according to the progression of the lesion. A marker of endothelial dysfunction is the increased expression of intercellular adhesion molecule ICAM1. In this paper, an immunohistochemistry method was standardized for the adhesion molecule ICAM-1, and its expression was studied in healthy human arteries with atheromatous plaque. In samples of human arteries with atherosclerotic disease, the expression of ICAM-1 was observed to be increased, but was hardly recognizable. This mainly because the tissue used as a control for standardization was a tonsil with an inflammatory process and hyperplasia, which significantly increases the expression of ICAM-1. The implementation of the immunohistochemistry method for ICAM1 in human arteries will reveal endothelial dysfunction states that will enable a future design and implementation of methods of diagnosis in atherosclerotic processes in the early stages.

KEY WORDS: ICAM-1; Atherosclerosis; Immunohistochemistry. 
MOORE-CARRASCO, R.; DONOSO, W.; SUTIN, F.; VERGARA, M.; TOLOZA, L.; GONZALEZ, J.; MORALES, E. \& PALOMO, I. Expresión de ICAM-1 en el endotelio de arterias humanas mediante inmunohistoquímica. Int. J. Morphol., 29(4):1351-1356, 2011.

\section{REFERENCIAS BIBLIOGRÁFICAS}

Badimón, L.; Vilahur, G. \& Padró, T. Lipoproteínas, plaquetas y aterotrombosis. Rev. Esp. Cardiol., 62(10):1161-78, 2009.

Balkau, B.; Charles, M. A.; Drivsholm, T.; Borch-Johnsen, K.; Wareham, N.; Yudkin, J. S.; Morris, R.; Zavaroni, I.; van Dam, R.; Feskins, E.; Gabriel, R.; Diet, M.; Nilsson, P.; Hedblad, B. \& European Group For The Study Of Insulin Resistance (EGIR). Frequency of the WHO metabolic syndrome in European cohorts, and an alternative definition of an insulin resistance syndrome. Diabetes Metab., 28(5):364-76, 2002.

Bustos, P.; Amigo, H.; Arteaga, A.; Acosta, A. M. \& Rona, R. J. Risk factors of cardiovascular disease among young adults. Rev. Med. Chil., 131(9):973-80, 2003.

Calderón, J. C.; Fernández, A. Z. \& María de Jesús, A. I. Aterosclerosis, estrés oxidativo y actividad física. Revisión. Invest. Clín., 49(3):397-410, 2008.

Chatterjee, S. Sphingolipids in atherosclerosis and vascular biology. Arterioscler. Thromb. Vasc. Biol., 18(10):1523-33, 1998.

Cobb, R. R.; Felts, K. A.; Parry, G. C. \& Mackman, N. Proteasome inhibitors block VCAM-1 and ICAM-1 gene expression in endothelial cells without affecting nuclear translocation of nuclear factor-kappa B. Eur. J. Immunol., 26(4):839-45, 1996.

Cybulsky, M. I. \& Gimbrone, M. A. Jr. Endothelial expression of a mononuclear leukocyte adhesion molecule during atherogenesis. Science, 251(4995):788-91, 1991.

Dandona, P.; Aljada, A.; Chaudhuri, A.; Mohanty, P. \& Garg, R. Metabolic syndrome: a comprehensive perspective based on interactions between obesity, diabetes, and inflammation. Circulation, 111(11):1448-54, 2005.

Delgado-Gonzáles, V. \& Leyva-Sartori, M. Use of direct inmunofluorescency on connective tissue diseases. Dermatol. Perú, 16(2):148-50, 2006.

Fuster, V. \& Sanz, J. La resonancia magnética en la evaluación de la aterotrombosis, del miocardio isquémico, hibernado o cicatrizado y de la microcirculación. Rev. Esp. Cardiol., 58(2):1421, 2005.

Janeway, C. A.; Travers, P.; Walport, M. \& Shlomchik, M. Immunobiology, the Immune System in Health and Disease. 6th Ed. New York, Churchill Livingstone, 2004.

Kiernan, J. A. Histological and histochemical methods. Theory and practice. $4^{\text {th }}$ Ed. Bloxham, UK, Scion, 2008.

Kurz, K. D.; Main, B. W. \& Sandusky, G. E. Rat model of arterial thrombosis induced by ferric chloride. Thromb. Res., 60(4):269-80, 1990.
Lehmann, J. C.; Jablonski-Westrich, D.; Haubold, U.; GutierrezRamos, J. C.; Springer, T. \& Hamann, A. Overlapping and selective roles of endothelial intercellular adhesion molecule-1 (ICAM-1) and ICAM-2 in lymphocyte trafficking. J. Immunol., 171(5):2588-93, 2003.

Lovett, J. K.; Gallagher, P. J.; Hands, L. J.; Walton, J. \& Rothwell, P. M. Histological correlates of carotid plaque surface morphology on lumen contrast imaging. Circulation, 110(15):2190-7, 2004.

Palomo, I.; Alarcón, M.; Moore-Carrasco, R. \& Argilés, J. M. Hemostasis alterations in metabolic syndrome (review). Int. J. Mol. Med., 18(5):969-74, 2006.

Palomo, I. G.; Gutiérrez, C. L.; Alarcón, M. L.; Jaramillo, J. C.; Segovia, F. M.; Leiva, E. M.; Mujica, V. E.; Icaza, G. N.; Díaz, N. S. \& Moore-Carrasco, R. Increased concentration of plasminogen activator inhibitor-1 and fibrinogen in individuals with metabolic syndrome. Mol. Med. Report, 2(2):253-7, 2009.

Palomo, I.; Moore-Carrasco, R.; Alarcon, M.; Rojas, A.; Espana, F.; Andres, V. \& Gonzalez-Navarro, H. Pathophysiology of the proatherothrombotic state in the metabolic syndrome. Front. Biosci. (Schol. Ed.), 2:194-208, 2010.

Stary, H. C.; Chandler, A. B.; Dinsmore, R. E.; Fuster, V.; Glagov, S.; Insull, W. Jr.; Rosenfeld, M. E.; Schwartz, C. J.; Wagner, W. D. \& Wissler, R. W. A definition of advanced types of atherosclerotic lesions and a histological classification of atherosclerosis. A report from the Committee on Vascular Lesions of the Council on Arteriosclerosis, American Heart Association. Arterioscler. Thromb. Vasc. Biol., 15(9):1512-31, 1995.

Velarde, M. S.; Prado, M. M.; Carrizo, T. R.; Abregú, A. V. \& Sánchez, S. S. Evaluación de disfunción endotelial en pacientes con diabetes tipo 2. Acta Bioquím. Clín. Latinoam., 41(4):491-7. 2007.

Viganò, P.; Infantino, M.; Lattuada, D.; Lauletta, R.; Ponti, E.; Somigliana, E.; Vignali, M. \& DiBlasio, A. M. Intercellular adhesion molecule-1 (ICAM-1) gene polymorphisms in endometriosis. Mol. Hum. Reprod., 9(1):47-52, 2003.

Wertheimer, S. J.; Myers, C. L.; Wallace, R. W. \& Parks, T. P. Intercellular adhesion molecule-1 gene expression in human endothelial cells. Differential regulation by tumor necrosis factor-alpha and phorbol myristate acetate. J. Biol. Chem., 267(17):12030-5, 1992.

Dirección para correspondencia:

Prof. Dr. Rodrigo Moore-Carrasco

Departamento de Bioquímica Clínica e Inmunohematología Facultad de Ciencias de la Salud, Universidad de Talca

P.O. Box: 747, Talca

CHILE

Email: rmoore@utalca.cl

Recibido : 31-08-2011

Aceptado: 02-10-2011 